\title{
Outcome of Patients with HER2-Positive Advanced Breast Cancer Progressing During Trastuzumab-Based Therapy
}

\author{
Filippo Montemurro, ${ }^{\mathrm{a}}$ Michela Donadio, ${ }^{\mathrm{b}}$ Matteo Clavarezza, ${ }^{\mathrm{c}}$ Stefania Redana, ${ }^{\mathrm{a}}$ \\ Maria Elena Jacomuzzi, ${ }^{\mathrm{d}}$ Giorgio Valabrega, ${ }^{\mathrm{a}}$ Saverio Danese, ${ }^{\mathrm{e}}$ Guido Vietti-Ramus, ${ }^{\mathrm{f}}$ \\ Antonio Durando, ${ }^{\mathrm{g}}$ Marco Venturini, ${ }^{\mathrm{c}}$ Massimo Aglietta ${ }^{\mathrm{a}}$
}

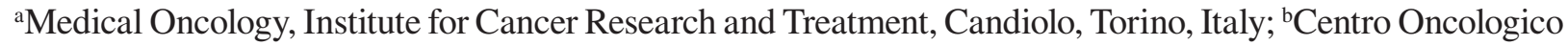
Subalpino (COES), Molinette Hospital, Torino, Italy; 'Senology Disease Management Team, Istituto Nazionale Tumori, Genova, Italy; ${ }^{\mathrm{d}}$ Gynaecologic Oncology, Mauriziano Hospital, Torino, Italy; ${ }^{\mathrm{e}}$ Gynaecology, S. Anna Hospital, Torino, Italy; ${ }^{\mathrm{f} I n t e r n a l ~ M e d i c i n e, ~ G i o v a n n i ~ B o s c o ~ H o s p i t a l, ~ T o r i n o, ~ I t a l y ; ~}{ }^{\mathrm{g} C l i n i c a}$ Universitaria, S. Anna Hospital, Torino, Italy

Key Words. HER2 • Metastatic breast cancer • Survival • Time to progression • Trastuzumab - Tumor progression

\begin{abstract}
We sought to describe patterns of treatment and clinical outcome in patients with HER2-positive advanced breast cancer progressing on trastuzumab-based therapy. One hundred eighty-four consecutive HER2-positive advanced breast cancer patients received trastuzumab-based therapy between September 1999 and September 2004. Patients were followed up until death or May 2005. For patients progressing on trastuzumabbased therapy, we calculated the response rate $(\mathrm{RR})$ to the first post-progression treatment, overall survival (OS) from the first administration of trastuzumab, time to second progression (TT-SP), and post-progression survival (PPS), according to treatment. At the time of this analysis, 132 patients had progressed on trastuzumab-based therapy, and 89 had died. Of the progressing patients, 21 experienced rapid progression and could not receive additional anticancer treatments; 40 patients
\end{abstract}

continued trastuzumab either alone (12 patients with isolated central nervous system progression), with chemotherapy ( 23 patients), or with endocrine therapy ( 5 patients); and 71 stopped trastuzumab and received chemotherapy (61 patients) or endocrine therapy (10 patients) as the first post-progression treatment. Excluding patients with rapid progression, clinical outcomes were similar whether trastuzumab was continued or not, in terms of RR $(18 \%$ and $27 \%$, respectively), OS (31 and 30 months, respectively), TT-SP (6 and 7 months, respectively), and PPS (21 and 19 months, respectively). The clinical outcome of patients with HER2-positive advanced breast cancer progressing during trastuzumab-based therapy might not be influenced by continuing trastuzumab. The optimal therapeutic strategy in this setting of patients needs evaluation in randomized trials. The Oncologist 2006;11:318-324

\section{INTRODUCTION}

Trastuzumab, a monoclonal antibody directed against HER2, the product of the c-erbB-2 proto-oncogene, has represented a major improvement in the treatment of the subset of 20\%-30\% human breast cancers with this genetic abnormality. Randomized studies in the metastatic and adjuvant settings have shown that the addition of trastuzumab to chemotherapy results in significant improvements in clinical

Correspondence: Filippo Montemurro, M.D., Medical Oncology, Institute for Cancer Research and Treatment, IRCC Candiolo, Strada Provinciale 142, 10060 Candiolo, Torino, Italy. Telephone: 39-011-9933250; Fax: 39-011-9933275; e-mail: fmontemurro@ircc. mauriziano.it Received November 18, 2006; accepted for publication February 27, 2006. CAlphaMed Press 1083-7159/2006/\$20.00/0 
outcomes, including overall survival [1-4]. Yet, because of its nature of targeted therapy and the uncertainties on its exact mechanism of action, several issues, which have relevant clinical and pharmacoeconomic implications, remain unresolved. Trastuzumab as a single agent has a response rate that peaks at around 35\% in appropriately selected metastatic breast cancer patients [5]. When added to chemotherapy in the same setting, the response rate is almost double, and survival is longer than with chemotherapy alone $[1,2]$. Unfortunately, most of the metastatic patients, including those with an impressive tumor response, develop disease progression during treatment. Preclinical experiments showed that trastuzumab exerts a cytostatic, rather than a cytotoxic, action on HER2-positive breast cancer cells [6]. Its withdrawal results in cancer growth, which can be suppressed by reintroducing the monoclonal antibody. On the basis of these observations and on the results of the early clinical trials, there is consensus that, in a responding patient with metastatic breast cancer, trastuzumab should be continued at least until tumor progression. In contrast, whether to stop or continue trastuzumab in a patient showing disease progression while on treatment is a subject of controversy. Preclinical data demonstrated that trastuzumab has additive or even synergistic effects with most of the cytostatic agents that are active in the treatment of breast cancer, including taxanes, vinorelbine, platinum salts, and others [7, 8]. These data, coupled with the tolerability of trastuzumab, have suggested to clinicians that continuing trastuzumab and changing the companion cytostatic agent could be a reasonable option in patients progressing on trastuzumab-based therapy. A few retrospective analyses in patients with HER2-positive advanced breast cancer showed encouraging response rates and survival when, upon disease progression, trastuzumab was continued and patients received further chemotherapy [9-11]. One obvious flaw of these reports is that patients discontinuing trastuzumab because of serious trastuzumab-related toxicity, or those experiencing rapid disease progression during the initial trastuzumab-based therapy and unable to receive further anticancer therapy, were not considered. Therefore, an encouraging clinical outcome might be the result of a selection bias. Indeed, in a follow-up analysis of 42 patients enrolled in a phase II trial of docetaxel and trastuzumab [12,13], we observed that progressing patients who could receive additional anticancer treatments without trastuzumab had clinical outcomes that were in the range of patients continuing trastuzumab beyond disease progression. In the absence of data from a randomized trial, our aim was to describe patterns of disease progression and treatment in patients with HER2-positive advanced breast cancer receiving trastuzumab-based therapy.

\section{Patients And Methods}

We collected information on 184 consecutive women with HER2-positive advanced breast cancer who received trastuzumab-based therapy between September 1999 and September 2004 at seven Italian institutions. Patient characteristics are summarized in Table 1. Data were entered into a database, and follow-up information was updated as of May 2005. Data on the initial trastuzumab-based regimens are summarized in Table 2. Sixty patients took part in phase II clinical trials evaluating trastuzumab in association with docetaxel (42 patients), with docetaxel and epirubicin (14 patients), and with docetaxel and liposomal doxorubicin (4 patients), whereas the other 124 received trastuzumab-based therapy outside clinical trials. Response rates to the initial trastuzumab-based regimen (complete response [CR] plus partial response [PR]) were $55.4 \%$ (95\% confidence interval [CI], 48.2\%-62.4\%), 57.4\% (95\% CI, $49.4 \%-65.1 \%$ ), and $71.0 \%$ (95\% CI, $59.4 \%-80.4 \%$ ) in the overall population, in patients with strong immunohistochemical (IHC) positivity for HER2 (3+), and in patients with HER2-positive tumors by fluorescence in situ hybridization (FISH), respectively. The median time to progression was 9.0 months (95\% CI, 7.4-11.5 months). Details of the first post-progression treatment were required, but investigators were invited to provide information on any further anticancer treatment the patient received until death or the end of follow-up.

Table 1. Patient characteristics

\begin{tabular}{|c|c|}
\hline Variable & Value \\
\hline Median age in years (range) & $53(29-80)$ \\
\hline Median disease-free interval ${ }^{\mathrm{a}}$ & $21(0-752)$ \\
\hline \multicolumn{2}{|l|}{ HER2 status } \\
\hline IHC $3+$ & $148(80 \%)$ \\
\hline IHC $2+$ & $18(10 \%)$ \\
\hline IHC positive NAS & $18(10 \%)$ \\
\hline ER-and/or PgR-positive & $93(50 \%)$ \\
\hline Prior exposure to anthracycline & $128(70 \%)$ \\
\hline Prior exposure to taxane & $67(36 \%)$ \\
\hline Up-front metastatic presentation & $38(21 \%)$ \\
\hline $\begin{array}{l}\text { Received T-based treatment as first-line } \\
\text { therapy for metastases }\end{array}$ & $119(66 \%)$ \\
\hline Single metastatic site & $66(36 \%)$ \\
\hline Visceral involvement (liver and lung) & $137(74 \%)$ \\
\hline \multicolumn{2}{|c|}{$\begin{array}{l}\text { aMonths from surgery to the first evidence of recurrent } \\
\text { disease. } \\
\text { Abbreviations: ER, estrogen receptor; FISH, fluorescence in } \\
\text { situ hybridization; IHC, immunohistochemistry; NAS, Not } \\
\text { otherwise specified; PgR, progesterone receptor; T, trastu- } \\
\text { zumab. }\end{array}$} \\
\hline
\end{tabular}




\section{Statistical Analyses}

In patients who progressed during the initial trastuzumabbased regimen, we studied the following outcomes: (a) response rate (RR) to the first post-progression treatment (CR plus PR), as reported by each investigator at each site; (b) overall survival (OS), which was calculated from the day of the first administration of trastuzumab-based therapy to the documented date of death; (c) time-to-second tumor progression (TT-SP), which was calculated from the date of the first progression on trastuzumab-based therapy to the date of documented further tumor progression, or death of the patient in the absence of tumor progression; (d) post-progression survival (PPS), which was calculated from the date of the first progression on trastuzumab-based therapy to the date of the documented death of the patient. Patients alive were censored at the date of the last follow-up contact. Survival analyses were conducted by the KaplanMeier method. Because of the retrospective nature of the analysis and the descriptive aim of this report, we did not perform statistical comparisons of the clinical outcomes in different patient subsets.

\section{RESULTS}

Figure 1 shows the flowchart of events and patterns of treatment for the entire group of 184 patients. The median follow-up for all the patients was 17 months (range, 1-66 months). Eighteen patients (10\%) discontinued trastuzumab in the absence of tumor progression either because of unacceptable toxicity or the patient's or treating physi-

Table 2. Summary of initial trastuzumab-based regimens

\begin{tabular}{|c|c|c|}
\hline Treatment & $\begin{array}{l}\text { No. of } \\
\text { patients }\end{array}$ & $\%$ \\
\hline Docetaxel, $75 \mathrm{mg} / \mathrm{m}^{2} \mathrm{q} 3 \mathrm{w}$, plus T & 78 & 42 \\
\hline Vinorelbine, $25 / 30 \mathrm{mg} / \mathrm{m}^{2}$ weekly, plus T & 43 & 23 \\
\hline $\begin{array}{l}\text { Docetaxel, } 75 \mathrm{mg} / \mathrm{m}^{2} \text {, plus epirubicin, } 75 \\
\mathrm{mg} / \mathrm{m}^{2} \text {, q3w plus T }\end{array}$ & 14 & 8 \\
\hline Paclitaxel, 80/90 mg/m² weekly, plus T & 12 & 6 \\
\hline Paclitaxel, $175 \mathrm{mg} / \mathrm{m}^{2} \mathrm{q} 3 \mathrm{w}$, plus T & 4 & 2 \\
\hline 5-FU CI plus vinorelbine plus T & 7 & 4 \\
\hline 5-FU CI plus T & 3 & 2 \\
\hline $\begin{array}{l}\text { Paclitaxel, } 80 / 90 \mathrm{mg} / \mathrm{m}^{2} \text { weekly, plus car- } \\
\text { boplatin, AUC } 2 \text { weekly, plus T }\end{array}$ & 4 & 2 \\
\hline $\begin{array}{l}\text { Docetaxel, } 75 \mathrm{mg} / \mathrm{m}^{2} \text {, plus liposomal } \\
\text { doxorubicin, } 50 \mathrm{mg} / \mathrm{m}^{2} \mathrm{q} 3 \mathrm{w} \text {, plus T }\end{array}$ & 4 & 2 \\
\hline Other regimens plus $\mathrm{T}$ & 8 & 4 \\
\hline Trastuzumab alone & 4 & 2 \\
\hline Unknown & 3 & 2 \\
\hline
\end{tabular}

Abbreviations: 5-FU, 5-fluorouracil; AUC, area under the concentration-versus-time curve; $\mathrm{CI}$, continuous infusion; q3w, every 3 weeks; T, trastuzumab. cian's decision (median duration of trastuzumab therapy, 7 weeks; range, 1-155 weeks). A total of 132 patients progressed while on the initial trastuzumab-based regimen and 89 died of tumor progression. Notably, a subset of 21 (16\%) patients experienced rapid progression and could receive only supportive care until death. Upon first progression, trastuzumab was continued in 40 and stopped in 71 patients (Table 3 and Table 4). In the former group, 12 patients with isolated central nervous system (CNS) progression received radiotherapy and continued trastuzumab alone. The other 28 received trastuzumab combined with chemotherapy (23 patients) or endocrine therapy (5 patients). In the latter group, 61 women received chemotherapy and 10 received

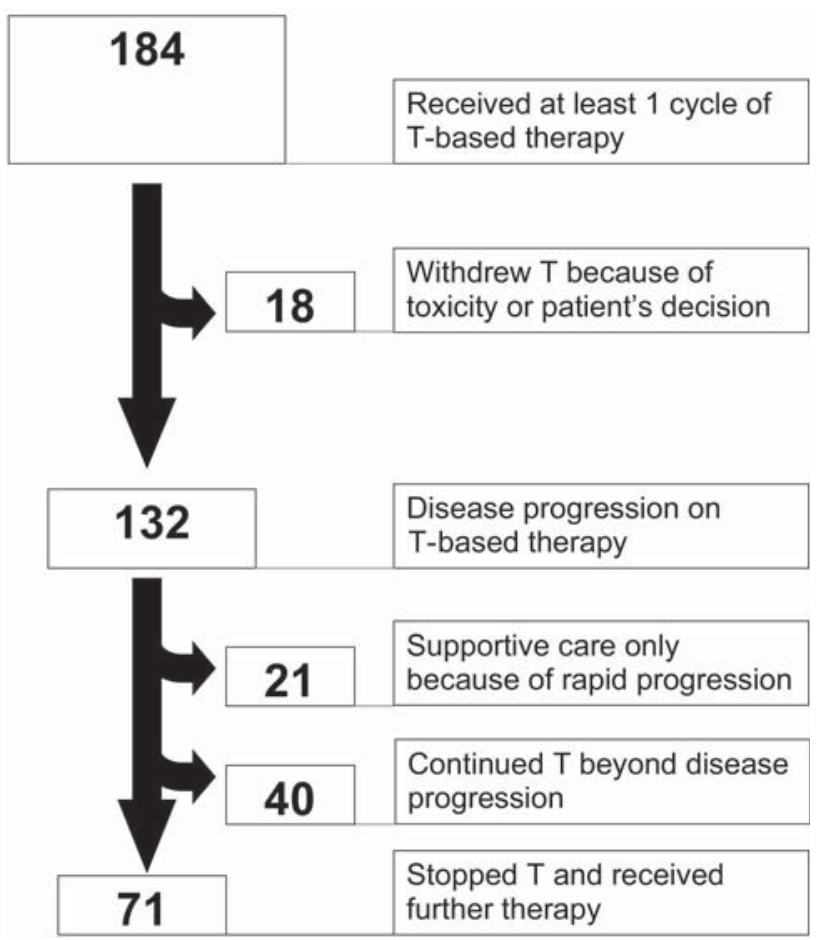

Figure 1. Flowchart of events. Abbreviation: T, trastuzumab.

Table 3. Summary of first post-progression treatments: patients continuing trastuzumab

\begin{tabular}{cl}
\hline Treatment (in addition to trastuzumab) & $\boldsymbol{n}$ \\
\hline Chemotherapy & 23 \\
Vinorelbine & 17 \\
Taxanes (docetaxel or paclitaxel) & 4 \\
Liposomal doxorubicin & 1 \\
Capecitabine & 1 \\
Endocrine therapy & 5 \\
Letrozole & 3 \\
Tamoxifen & 2 \\
Trastuzumab alone & 12 \\
\hline
\end{tabular}


endocrine therapy as the first post-progression treatment. Table 5 and Figure 2 summarize the clinical outcomes of patients according to the pattern of post-progression treatment. Women with isolated CNS progression were excluded from the calculation of RR. Survival outcomes were similar when we focused on patients receiving chemotherapy alone or chemotherapy with trastuzumab beyond progression, thus excluding patients who received endocrine therapy or those with isolated CNS metastases who received trastuzumab alone (data not shown). We conducted additional exploratory analyses for the subsets of patients with HER2overexpressing (HER2 3+ by IHC) and HER2-amplified tumors, with results that were similar to those of the whole group (data not shown). Additionally, we evaluated differ-

Table 4. Summary of first post-progression treatments: patients stopping trastuzumab

\begin{tabular}{ll}
\hline Treatment & $\boldsymbol{n}$ \\
\hline Chemotherapy & 61 \\
Vinorelbine & 11 \\
Taxanes (docetaxel or paclitaxel) & 9 \\
CMF-like regimens & 9 \\
Vinorelbine plus 5-FU CI & 6 \\
Anthracycline-based regimens & 6 \\
Vinorelbine plus gemcitabine & 5 \\
Capecitabine & 5 \\
Capecitabine plus cisplatin & 4 \\
Gemcitabine & 2 \\
Other regimens & 4 \\
Endocrine therapy & 10 \\
Letrozole & 7 \\
Exemestane & 2 \\
Megestrol acetate & 1 \\
\hline
\end{tabular}

Abbreviations: 5-FU, 5-fluorouracil; CI, continuous infusion; CMF, cyclophosphamide, methotrexate, 5-fluorouracil. ences in clinical characteristics of women continuing or stopping trastuzumab upon disease progression and found that the former were significantly younger (median age, 48 vs. 55 years; $p=.05$ ), were more likely to have responded (CR or PR) to the initial trastuzumab-based regimen (67\% vs. $52 \% ; p=.09$ ), and were more likely to have experienced isolated CNS progression during the initial trastuzumabbased regimen $(27 \%$ vs. $3 \% ; p<.01)$ than the latter. Other clinical characteristics, such as prior exposure to anthracyclines, pattern of metastatic disease, percentage of patients receiving trastuzumab-based therapy as first-line therapy for metastatic disease, and duration of response to the first trastuzumab-containing regimen, were similar between the two groups (data not shown).

\section{Discussion}

Our retrospective analysis results in HER2-positive advanced breast cancer patients progressing during trastuzumab-based therapy suggest that clinical outcome was similar, regardless of whether trastuzumab was continued beyond progression. Three papers reported on retrospective analyses focusing on patients continuing trastuzumab beyond disease progression [9-11]. Those studies included patients who had received at least two subsequent trastuzumab-based regimens for metastatic disease. Fountzilas et al. [10] described the clinical outcome of 80 patients continuing trastuzumab beyond disease progression in combination with additional chemotherapy. The first postprogression treatment resulted in a $24 \%$ response rate and a median progression-free survival duration of 5.2 months, but tumor responses were also seen with subsequent trastuzumab-based lines of treatment [10]. In a similar retrospective analysis conducted in 105 patients, Gelmon et al. [9] reported a $32 \%$ response rate and a median progression-free survival of 24-30.5 weeks when either trastuzumab alone or combined with chemotherapy was administered as second-

Table 5. Summary of clinical outcome beyond progression

\begin{tabular}{llll}
\hline & Rapidly progressing & Continuing trastuzumab & Stopping trastuzumab \\
\cline { 2 - 4 } Clinical outcome variable & $(\boldsymbol{n = 2 1 )}$ & $(\boldsymbol{n = 4 0})$ & $(\boldsymbol{n}=\mathbf{7 1})$ \\
\hline RR,\% & $\mathrm{NE}$ & $17.9(28)$ & $27.3(66)$ \\
$95 \%$ CI & $\mathrm{NE}$ & $7.9-35.6$ & $18.0-39.0$ \\
Median OS, months & 7.4 & 30.1 & 30.2 \\
$95 \%$ CI & $4.6-10.3$ & $26.9-33.3$ & $20.5-39.9$ \\
Median TT-SP, months & & 6.3 & 7.2 \\
$95 \%$ CI & $\mathrm{NE}$ & $5.0-7.6$ & $6.4-7.9$ \\
Median PPS, months & 2.4 & 21.0 & 18.7 \\
$95 \%$ CI & $1.9-2.9$ & $8.9-33.1$ & $12.3-25.0$ \\
\hline
\end{tabular}

${ }^{a}$ The numbers in parentheses indicate patients available for response evaluation.

Abbreviations: CI, confidence interval; NE, not evaluable; OS, overall survival; PPS, post-progression survival; RR, response rate (complete response plus partial response); TT-SP, time to second progression. 


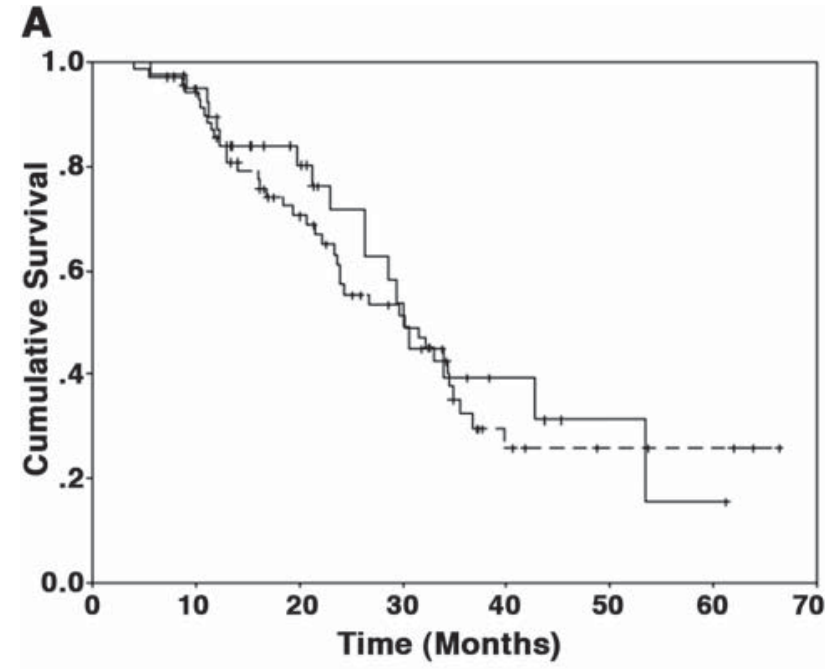

B

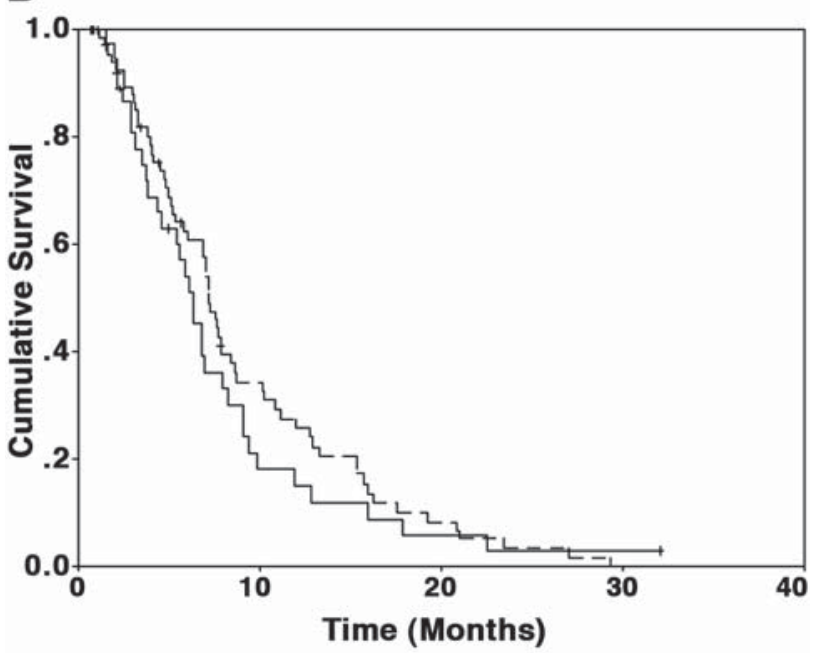

C

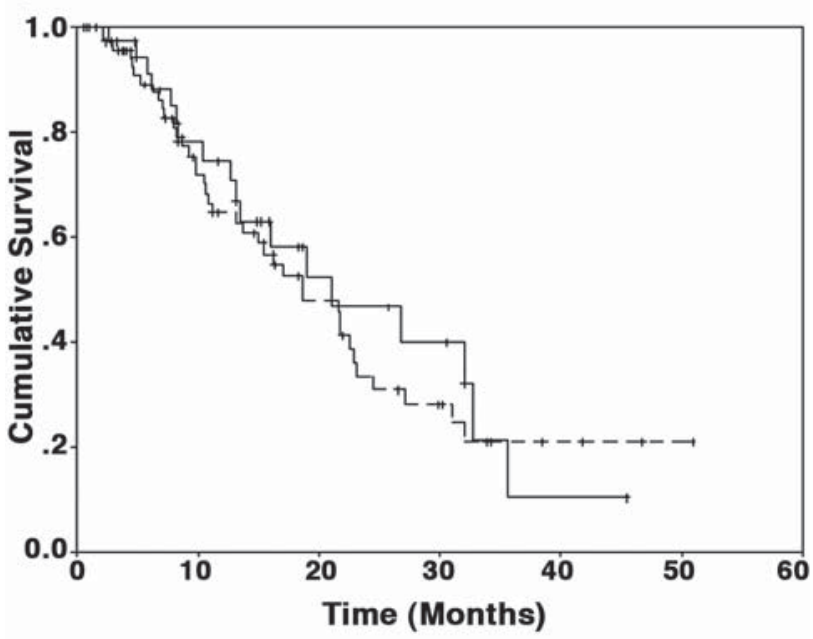

Figure 2. Kaplan-Meier estimates of overall survival (A), time to second progression (B), and post-progression survival (C) in patients receiving (dashed line) and not receiving (solid line) trastuzumab beyond disease progression. line treatment after failure of an initial trastuzumab-based regimen. The third paper reported the results of a companion treatment-extension trial of the $\mathrm{H} 0648 \mathrm{~g}$ pivotal randomized study of chemotherapy alone and chemotherapy plus trastuzumab in HER2-positive advanced breast cancer patients $[1,11]$. This companion study allowed patients in the chemotherapy-alone arms to receive trastuzumab upon disease progression and patients in the chemotherapy plus trastuzumab arms to continue trastuzumab beyond disease progression. While the primary aim of the companion study was to assess the safety of trastuzumab administered for long periods of time, the authors also reported efficacy results. In 93 patients continuing trastuzumab beyond disease progression, the response rate was $11 \%$, and the median duration of response was 6.7 months.

Although all authors placed caution on the interpretation of these outcomes, their results probably supported the practice of continuing trastuzumab beyond progression. Indeed, a recent longitudinal cohort study showed that, in the U.S., as many as $76 \%$ of patients progressing during an initial trastuzumab-based regimen received trastuzumab plus another chemotherapy in the following line of chemotherapy outside clinical trials [14].

Our paper and the others that we have discussed above share the common limitations of retrospective analyses. This approach cannot account for the selection bias that goes into individualized therapeutic decisions. For example, we found that women who continued trastuzumab beyond progression were younger and more likely to have achieved tumor response to the initial trastuzumab-containing regimen compared with patients stopping trastuzumab upon disease progression. This may reflect different attitudes of physicians toward particular subsets of patients, making comparisons between outcomes of patients receiving different treatments unreliable. Another limitation of our analysis comes from the long half-life of trastuzumab once the steady state has been reached [14]. As a consequence, even in patients discontinuing trastuzumab and receiving different anticancer agents, tumor response may still depend on potential additive or synergistic effects of the monoclonal antibody. With these limitations well in mind, however, we believe that our data provide some valuable information for the clinician, as well as implications for further research. First, chemotherapy without trastuzumab yielded reassuring results in terms of response rate and survival in patients who progressed on trastuzumab-based therapy. Breast cancer with HER2 amplification or overexpression has long been recognized as a biologically aggressive disease $[15,16]$. Trastuzumabbased treatments have improved the prognosis for HER2positive breast cancer, to the point that it might become 
better than that of HER2-negative tumors treated with the same chemotherapy [17]. It is thus commonly perceived that, after trastuzumab failure, HER2-positive metastatic breast cancer patients have few options left. However, our data show that, with the exception of patients with rapidly progressing disease, chemotherapy and endocrine therapy were active in a significant proportion of patients, apparently regardless of trastuzumab continuation. Second, similar to cytotoxic chemotherapy or endocrine therapy, for which progression during treatment denotes "chemoresistant" or "endocrine-resistant" disease, tumor progression during trastuzumab is likely to be sustained by specific mechanisms of resistance to this agent. Results of the recently completed adjuvant randomized trials [3, 4] will translate into a greater number of patients receiving trastuzumab as part of the treatment for early HER2-positive disease. Resistance to this agent will therefore become a relevant issue when deciding the therapeutic conduct in those patients who develop metastatic disease during or shortly after adjuvant treatment with trastuzumab. Unfortunately, despite several interesting hypotheses having been generated in preclinical and translational studies [18, 19], resistance to trastuzumab has not yet been defined in patients. Results with lapatinib, a small-molecule dual inhibitor of both epidermal growth factor receptor 1 and HER2, in heavily pretreated patients with HER2-positive advanced breast cancer who had failed prior trastuzumabbased treatments [20] further confirm that resistance to trastuzumab develops in patients and that it could be potentially overcome by other targeting agents.

Third, and not less important, trastuzumab therapy is expensive [21]. Outside the use as first-line therapy in appropriately selected HER2-positive breast cancer patients, its cost-effectiveness is unknown and probably unfavorable.

Many authorities in the field of breast cancer have invoked appropriately designed randomized trials to define the optimal therapeutic approach in women with HER2positive advanced breast cancer developing disease progression while on trastuzumab-based therapy. These trials should clarify clinical as well as biological and pharmaeco- nomic issues. Unfortunately, so far, the few initiatives that have been launched have failed to reach their goals because of insufficient accrual and possibly also because of patients' and doctors' biases toward continuing trastuzumab beyond progression [22]. At present, we are aware of only two active large trials that are randomizing patients progressing while on trastuzumab-based therapy to capecitabine alone or capecitabine plus trastuzumab [23] and to vinorelbine alone or vinorelbine plus trastuzumab [24].

\section{Conclusion}

In summary, we showed that further lines of chemotherapy or endocrine therapy without trastuzumab yielded acceptable rates of disease control in HER2-positive advanced breast cancer patients progressing on trastuzumab-based therapy. Our data question the policy of continuing trastuzumab beyond disease progression. However, as they were generated from retrospective analyses, neither data for nor against the continuation of trastuzumab beyond progression should translate into clinical guidelines at the present time. In our opinion, clinicians facing therapeutic decisions should consider and discuss with their patients that continuation of trastuzumab beyond disease progression has theoretical but unproven efficacy and that disease control can be achieved with other anticancer treatments, even discontinuing trastuzumab. Patient expectations should also be taken into account, as well as additional costs and rare but potentially severe cardiac trastuzumab-related toxicity.

Efforts should be focused on completing the accrual of the ongoing randomized trials and on planning new initiatives in order to establish the correct strategy in this setting of patients.

\section{ACKNOWLEDGMENT}

This work was in part supported by a grant from Regione Piemonte (Ricerca Sanitaria Finalizzata 2003).

\section{Disclosure of Potential Conflicts OF INTEREST}

The authors indicate no potential conflicts of interest.

\section{REFERENCES}

1 Slamon DJ, Leyland-Jones B, Shak S et al. Use of chemotherapy plus a monoclonal antibody against HER 2 for metastatic breast cancer that overexpresses HER2. N Eng1 J Med 2001;344:783-792.

2 Marty M, Cognetti F, Maraninchi D et al. Randomized phase II trial of the efficacy and safety of trastuzumab combined with docetaxel in patients with human epidermal growth factor receptor 2-positive metastatic breast cancer administered as first-line treatment: the M77001 study group. J Clin Oncol 2005;23:4265-4274.
3 Piccart-Gebhart MJ, Procter M, Leyland-Jones B et al. Trastuzumab after adjuvant chemotherapy in HER2-positive breast cancer. N Engl J Med 2005;353:1659-1672.

4 Romond EH, Perez EA, Bryant J et al. Trastuzumab plus adjuvant chemotherapy for operable HER2-positive breast cancer. N Eng1 J Med 2005;353:1673-1684.

5 Vogel CL, Cobleigh MA, Tripathy D et al. Efficacy and safety of trastuzumab as a single agent in first-line treatment of HER2-overexpressing metastatic breast cancer. J Clin Oncol 2002;20:719-726. 
6 Pietras RJ, Pegram MD, Finn RS et al. Remission of human breast cancer xenografts on therapy with humanized monoclonal antibody to HER-2 receptor and DNA-reactive drugs. Oncogene 1998;17:2235-2249.

7 Pegram M, Hsu S, Lewis G et al. Inhibitory effects of combinations of HER-2/neu antibody and chemotherapeutic agents used for treatment of human breast cancers. Oncogene 1999;18:2241-2251.

8 Pegram MD, Konecny GE, O'Callaghan C et al. Rational combinations of trastuzumab with chemotherapeutic drugs used in the treatment of breast cancer. J Natl Cancer Inst 2004;96:739-749.

9 Gelmon KA, Mackey J, Verma S et al. Use of trastuzumab beyond disease progression: observations from a retrospective review of case histories. Clin Breast Cancer 2004;5:52-58; discussion 59-62.

10 Fountzilas G, Razis E, Tsavdaridis D et al. Continuation of trastuzumab beyond disease progression is feasible and safe in patients with metastatic breast cancer: a retrospective analysis of 80 cases by the Hellenic Cooperative Oncology Group. Clin Breast Cancer 2003;4:120-125.

11 Tripathy D, Slamon DJ, Cobleigh M et al. Safety of treatment of metastatic breast cancer with trastuzumab beyond disease progression. J Clin Oncol 2004;22:1063-1070.

12 Montemurro F, Choa G, Faggiuolo R et al. A phase II study of threeweekly docetaxel and weekly trastuzumab in HER2-overexpressing advanced breast cancer. Oncology 2004;66:38-45.

13 Montemurro F, Faggiuolo R, Redana S et al. Continuation of trastuzumab beyond disease progression. J Clin Oncol 2005;23:2866-2868; discussion 2268-2269.

14 Leyland-Jones B, Arnold A, Gelmon K et al. Pharmacologic insights into the future of trastuzumab. Ann Oncol 2001;12(suppl 1):S43-S47.

15 Slamon DJ, Clark GM, Wong SG et al. Human breast cancer: correlation of relapse and survival with amplification of the HER-2/neu oncogene. Science 1987;235:177-182.
16 Kim YS, Konoplev SN, Montemurro Fet al. HER-2/neu overexpression as a poor prognostic factor for patients with metastatic breast cancer undergoing high-dose chemotherapy with autologous stem cell transplantation. Clin Cancer Res 2001;7:4008-4012.

17 Papaldo P, Fabi A, Ferretti G et al. A phase II study on metastatic breast cancer patients treated with weekly vinorelbine with or without trastuzumab according to HER2 expression: changing the natural history of HER2-positive disease. Ann Oncol 2006 Jan 12; [Epub ahead of print].

18 Nagata Y, Lan KH, Zhou X et al. PTEN activation contributes to tumor inhibition by trastuzumab, and loss of PTEN predicts trastuzumab resistance in patients. Cancer Cell 2004;6:117-127.

19 Valabrega G, Montemurro F, Sarotto I et al. TGFalpha expression impairs Trastuzumab-induced HER2 downregulation. Oncogene 2005;24: 3002-3010.

20 Burris HA 3rd. Dual kinase inhibition in the treatment of breast cancer: initial experience with the EGFR/ErbB-2 inhibitor lapatinib. The Oncologist 2004;9(suppl 3):10-15.

21 Norum J, Risberg T, Olsen JA. A monoclonal antibody against HER-2 (trastuzumab) for metastatic breast cancer: a model-based cost-effectiveness analysis. Ann Oncol 2005;16:909-914.

22 Hortobagyi GN. Continuation of trastuzumab beyond disease progression. J Clin Oncol 2005;23:2868-2869.

23 German Breast Cancer Group. Herceptin TBP. [German]. Available at http://www.germanbreastgroup.de/herceptin/. Accessed November $11,2005$.

24 National Cancer Institute. Phase III Randomized Study of Vinorelbine With or Without Trastuzumab (Herceptin $®)$ in Women With HER2-Positive Progressive Metastatic Breast Cancer. SWOG-S0347, NCT00103233. Available at http://www.cancer.gov/search/ViewClinicalTrials.aspx?cdr id $=409573 \&$ version $=$ HealthProfessional $\&$ protocolsearchid $=1800206$. Accessed November 11, 2005. 


\section{Outcome of Patients with HER2-Positive Advanced Breast Cancer Progressing During}

Trastuzumab-Based Therapy

Filippo Montemurro, Michela Donadio, Matteo Clavarezza, Stefania Redana, Maria Elena Jacomuzzi, Giorgio Valabrega, Saverio Danese, Guido Vietti-Ramus, Antonio Durando, Marco Venturini and Massimo Aglietta

The Oncologist 2006;11;318-324;

DOI: $10.1634 /$ theoncologist.11-4-318

This information is current as of May 14, 2012

Updated Information \& Services including high-resolution figures, can be found at: http://theoncologist.alphamedpress.org/content/11/4/318

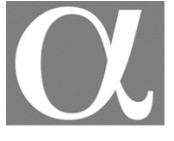

